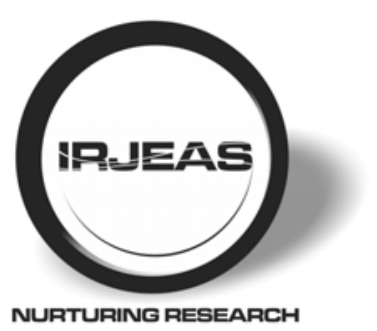

INTERNATIONAL RESEARCH JOURNAL

OF

ENGINEERING \& APPLIED SCIENCES

ISSN: 2322-0821(0)

VOLUME 9 ISSUE 3

ISSN: 2394-9910(P)

July 2021 - Sep 2021

www.irjeas.org

\title{
GRAPHENE - A NEW AGE OF MATERIAL: A REVIEW
}

\author{
Greyson Martin ${ }^{1}$, Liam Tremblay ${ }^{2}$ \\ ${ }^{1,2}$ Department of Material Science \& Engineering, Western University, Ontario, Canada \\ *Corr. Author - tremb.liam96@gmail.com
}

\begin{abstract}
We all be familiar with two well-known forms of Carbon which are diamond and graphite. Diamond is a one of the toughest and hard material in the earth. Graphite is well-known as most brittle material. But one thing had changed forever, when two physicists A.K.Geim and K.S. Novoselov had got noble prize for "Graphene". On the basis of his research work published title" The rise of Graphene". His work shows that Graphene is 200 times stronger than cast iron. It have so many desirable qualities to use in our daily life. Graphene, becoming eminent as a true 2-D material, had got ever-increasing attention due to its unmatched physicochemical properties (excellent conductivity, high surface area, high mechanical strength, ease of functionalization and mass production). Graphene is a extraordinarily amazing material with countless highest extents to its name. It is the thinnest material in the universe and the strongest yet calculated. Its charge carriers exhibit massive inherent mobility, have the negligible effective mass (it is zero) and can travel micrometer-long distances without scattering at room temperature. Graphene be able to uphold current densities 6 orders higher than copper, shows record thermal conductivity and stiffness, is impermeable to gases and reconciles such contradictory qualities as brittleness and ductility. Electron transport in graphene is described by a Dirac-like equation.
\end{abstract}

Graphene is a rapidly rising star on the horizon of materials science and condensed matter physics. This strictly two-dimensional material exhibits exceptionally high crystal and electronic quality and, despite its short history, has already revealed a cornucopia of new physics and potential applications, which are briefly discussed here. Whereas one can be certain of the realness of applications only when commercial products appear, graphene no longer requires any further proof of its importance in terms of fundamental physics. Owing to its unusual electronic spectrum, graphene has led to the emergence of a new paradigm of "relativistic" condensed matter physics, where quantum relativistic phenomena, some of which are unobservable in high energy physics, can now be mimicked and tested in table-top experiments. More generally, graphene represents a conceptually new class of materials that are only one atom thick and, on this basis, offers new inroads into low-dimensional physics that has never ceased to surprise and continues to provide a fertile ground for applications. This paper selectively reviews recent advancements in graphene.

Keywords- Carbon, Graphite, 2 -Dimensional, Honey Comb Structure, Single Layer Etc

\section{INTRODUCTION}

A crystalline form of carbon is known as graphene, beside among graphite, diamond, carbon nano-tubes and fullerenes. In Graphene, carbon atoms have set in an uniform hexagonal pattern. Graphene can be known as a one-atom thick layer of the layered mineral graphite. High quality graphene is very light,strong, nearly transparent, and an excellent conductor of electricity and heat . Its alloy with other materials and with light, and its inherently 2D nature, produce unique properties. During separation from graphite, many researchers studying carbon nano tubes were already well familiar with the composition, structure and properties of graphene, which had been calculated few decades earlier. The combination of familiarity, wonderful properties and surprising ease of isolation enabled an explosion in graphene research. Konstantin Novoselov and Andre Geim at the University of Manchester won the Nobel Prize in Physics in 2010 "for groundbreaking experiments regarding the two dimensional material graphene".[1]

\section{DESCRIPTION}

One an allotrope of carbon is graphene whose configuration is a single planar sheet of sp2-bonded C atoms, that are dense manner packed in a honeycomb crystal lattice structure.[2][4] Graphene is most easily visualized as an atomic-scale chicken wire made of carbon atoms and their bonds. The $\mathrm{C}-\mathrm{C}$ bond length in graphene is about $0.142 \mathrm{~nm}$.[5] Graphene sheets stack to form graphite with an inter planar area of $0.335 \mathrm{~nm}$. The IUPAC compendium of technology states: such as carbon sheets, carbon layers, or graphite layers have been used for the term graphene. it is incorrect to use for a single layer a term which includes the term graphite, which would imply a 3-D structure. The term graphene should be used only when the reactions, structural relations or other properties of individual layers.[6] In this regard, graphene has been referred to as an infinite alternant (only six-member carbon ring) polycyclic aromatic hydrocarbon (PAH). 
The biggest known isolated molecule of this type consists of 222 atoms and is 10 benzene rings across.[7] It has proven very difficult to formation even slightly bigger molecules, and they still remain "a dream of many organic and polymer chemists and scientists ".[8] Graphene sheet is thermodynamically unpredictable with respect to other fullerene structures if its size is less than about $20 \mathrm{~nm}$ and becomes the most stable one only for sizes larger than 24,000 carbon atoms. [9] The flat graphene sheet is also known to be unstable with respect to scrolling i.e. curling up, which is its lower energy state.[10]

A definition of "graphene is a one atomic plane of graphite. This definition is narrower than the definitions given above and refers to cleaved, transferred and suspended graphene mono layers. Other forms of graphene, such as graphene grown on various metals, can also become free-standing if, for example, suspended or transferred to silicon dioxide $\left(\mathrm{SiO}_{2}\right)$. A new example of isolated graphene is graphene on silicon carbide $(\mathrm{SiC})$ after its spontaneous with hydrogen.[12]

\section{OCCURRENCE}

AND

In essence, graphene is an isolated atomic plane of graphite. From this perspective, graphene has been known since the invention of X-ray crystallography. Graphene planes become even better separated in intercalated graphite compounds. In 2004, physicists at the University of Manchester and the Institute for Microelectronics Technology, Chernogolovka, Russia, first isolated individual graphene planes by using adhesive tape.[13] They also tested electronic properties of the obtained flakes and showed their unique properties.[14] In 2005 the same Manchester Geim group together with the Philip Kim group from Columbia University shows that quasi-particles in graphene were mass less Dirac fermions. These discoveries led to an explosion of interest in graphene. Since then, hundreds of researchers have happened in the area, resulting in an extensive search for relevant earlier papers. The Manchester researchers themselves published the first literature review.[2] They cite several papers in which graphene or ultra-thin graphitic layers were epitaxially grown on various substrates. Also, they note a number of pre-2004 reports in which intercalated graphite compounds were studied in a transmission electron microscope. In the latter case, researchers occasionally observed extremely thin graphitic flakes (an individual layers). An early detailed study on few-layer graphene dates back to 1962.[15] The earliest TEM graphic images of few-layer graphene were published by F. Vogt and G. Ruess in 1948.[16] In 1859 Benjamin Collins Brodie was aware of the highly lamellar structure of thermally reduced graphite oxide.[17] It was studied in detail by V. Kohlschütter and P. Haenni in 1918, who also described the properties of graphite oxide paper.[18] It is now well known that tiny fragments of graphene sheets are produced whenever graphite is abraded, such as when drawing a line with a pencil.[13] There was little interest in this graphitic residue before 2004/05 and, therefore, the discovery of graphene is often attributed to Andre Geim and colleagues[19] who introduced graphene in its modern incarnation.

Year 2008 The most expensive material one of on Earth was graphene produced by exfoliation, with a sample that can be placed at the cross section of a human hair costing more than $\$ 1,000$ as of April 2008 (about $\$ 100,000,000 / \mathrm{cm} 2)$.[13] Since then, exfoliation procedures have been scaled up, and now companies sell graphene in large quantities.[20][21] On the other hand, the price of epitaxial graphene on $\mathrm{SiC}$ is dominated by the substrate price, which is approximately $\$ 100 / \mathrm{cm} 2$ as of 2009 . Byung Hee Hong and his team in South Korea pioneered the synthesis of large-scale graphene films using chemical vapour deposition (CVD) on thin nickel layers, which triggered chemical researches toward the practical applications of graphene,[22][23] with wafer sizes up to 30 inches $(760 \mathrm{~mm})$ reported.[24]

In 2011 the Institute of Electronic Materials Technology and Department of Physics, Warsaw University announced a joint development of acquisition technology of large pieces of graphene with the best quality so far.[25][26] In the literature, specifically that of the surface science community, graphene has also been commonly referred to as monolayer graphite. This community has intensely studied epitaxial graphene on various surfaces (over 300 articles prior to 2004). In some cases, these graphene layers are coupled to the surfaces weakly enough (by Van der Waals forces) to retain the two dimensional electronic band structure of isolated graphene,[27][28] as also happens[14] with exfoliated graphene flakes with regard to $\mathrm{SiO} 2$. An example of weakly coupled epitaxial graphene is the one grown on $\mathrm{SiC}$ (see below).

\section{EXFOLIATED GRAPHENE}

In 2004, the Manchester group of colleges obtained graphene by micro-mechanical alleviation of graphite. They used adhesive tape to repeatedly split graphite crystals into increasingly thinner pieces. They may not have been the first to use this technique -- US Patent $6,667,100$, filed in 2002, describes the process in detail, applied to commercially available flexible expanded graphite sheet, to achieve a graphite thickness of 0.01 thousandth of an inch. The tape with attached optically transparent flakes was dissolved in acetone, and, after a few further steps, the flakes including mono layers were sedimented on a silicon wafer. Individual atomic planes were then hunted with an optical microscope. A year later, the researchers simplified the technique and started using dry deposition, avoiding the stage when graphene floated in a liquid. Relatively large crystallites (first, only a few micro metres in size but eventually larger than $1 \mathrm{~mm}$ and visible to the naked eye) were obtained with the technique. It is often referred to as a scotch tape or drawing method. The latter name appeared because the dry deposition resembles drawing with a piece of graphite.[29] The key for the success probably was the use of high-throughput visual recognition of graphene on a properly chosen substrate, which provides a small but noticeable optical contrast. The optical properties section below contains a photograph of what graphene looks like. The isolation of graphene led to the current research boom. Previously, free-standing atomic planes were often "presumed not to exist"[14] because they are thermodynamically unstable on a nanometer scale[9] and, if unsupported, have a tendency to scroll and buckle.[8] It is currently believed that intrinsic microscopic roughening. on the scale of $1 \mathrm{~nm}$ could be important for the stability of purely $2 \mathrm{D}$ crystals.[30]

There were a number of previous attempts to make atomically thin graphitic films by using exfoliation 
International Research Journal of Engineering \& Applied Sciences, IRJEAS www.irjeas.org, ISSN(O): 2322-0821, Volume 9 Issue 3, Jul 2021-Sep 2021, Page 10-15

techniques similar to the drawing method. Multilayer samples down to $10 \mathrm{~nm}$ in thickness were obtained.

These efforts were reviewed in 2007.[2] Furthermore, a couple of very old papers were recently unearthed[15] in which researchers tried to isolate graphene starting with intercalated compounds. These papers reported the observation of very thin graphitic fragments (possibly monolayers) by transmission electron microscopy. Neither of the earlier observations was sufficient to "spark the graphene gold rush", until the Science

paper did so by reporting not only macroscopic samples of extracted atomic planes but, importantly, their unusual properties such as the bipolar transistor effect, ballistic transport of charges, large quantum oscillations, etc. The discovery of such interesting qualities intrinsic to graphene gave an immediate boost to further research and several groups quickly repeated the initial result and moved further. These breakthroughs also helped to attract attention to other production techniques, such as epitaxial growth of ultrathin graphitic films. In particular, it has later been found that graphene monolayers grown on $\mathrm{SiC}$ and $\mathrm{Ir}$ are weakly coupled to these substrates (how weakly remains debated) and the graphene-substrate interaction can be passivated further.[12]

Not only graphene but also free-standing atomic planes of boron nitride, mica, dichalcogenides and complex oxides were obtained by using the drawing method.[31] Unlike graphene, the other $2 \mathrm{D}$ materials have so far attracted surprisingly little attention.

\subsection{Epitaxial growth on silicon carbide}

Another method of obtaining graphene is to heat silicon carbide $(\mathrm{SiC})$ to high temperatures $\left(>1,100^{\circ} \mathrm{C}\right)$ under lower pressure $(\sim 10-6$ torr) to reduce it to graphene.[32] This process produces epitaxial graphene with dimensions dependent upon the size of the SiC substrate (wafer). The face of the $\mathrm{SiC}$ used for graphene formation, silicon- or carbon-terminated, highly influences the thickness, mobility and carrier density of the graphene. Many important graphene properties have been identified in graphene produced by this method. For example, the electronic band-structure (so-called Dirac cone structure) has been first visualized in this material.[33][34][35] Weak anti-localization is observed in this material and not in exfoliated graphene produced by the pencil-trace method.[36] Extremely large, temperature-independent mobilities have been observed in SiC-epitaxial graphene. They approach those in exfoliated graphene placed on silicon oxide but still much lower than mobilities in suspended graphene produced by the drawing method. It was recently shown that even without being transferred, graphene on $\mathrm{SiC}$ exhibits the properties of massless Dirac fermions such as the anomalous quantum Hall effect. [37][38][39][40][41] The weak vander Waals force that provides the cohesion of multilayer graphene stacks does not always affect the electronic properties of the individual graphene layers in the stack. That is, while the electronic properties of certain multilayered epitaxial graphenes are identical to that of a single graphene layer,[42] in other cases the properties are affected[33][34] as they are for graphene layers in bulk graphite. This effect is theoretically well understood and is related to the symmetry of the interlayer interactions.[42] Epitaxial graphene on $\mathrm{SiC}$ can be patterned using standard microelectronics methods. The possibility of large integrated electronics on $\mathrm{SiC}$ epitaxial graphene was first proposed in 2004,[43] and a patent for graphene-based electronics was filed provisionally in 2003 and issued in 2006.[44] Since then, important advances have been made. In 2008, researchers at MIT Lincoln Lab produced hundreds of transistors on a single chip[45] and in 2009 , very high frequency transistors were produced at the Hughes Research Laboratories on monolayer graphene on SiC.[46] Band gap of the epitaxial graphene can be tuned by irradiating with laser beams; modified graphene has a lot of advantages in device application.[47]

\section{a. Epitaxial growth on metal substrates}

This method uses source and the atomic structure of a metal substrate to seed the growth of the graphene (epitaxial growth). Graphene grown on ruthenium doesn't typically yield a sample with a uniform thickness of graphene layers, and bonding between the bottom graphene layer and the substrate may affect the properties of the carbon layers.[48] On the other hand, graphene grown on iridium is very weakly bonded, uniform in thickness, and can be made highly ordered. Like on many other substrates, graphene on iridium is slightly rippled. Due to the long-range order of these ripples, generation of minigaps in the electronic bandstructure (Dirac cone) becomes visible.[49] High-quality sheets of few-layer graphene exceeding $1 \mathrm{~cm} 2(0.2 \mathrm{sq}$ in) in area have been synthesized via chemical vapor deposition on thin nickel films with methane as a carbon source. These sheets have been successfully transferred to various substrates, demonstrating viability for numerous applications.[24][37] [50] An improvement of this technique has employed copper foil; at very low pressure, the growth of graphene automatically stops after a single graphene layer forms, and arbitrarily large graphene films can be created.[24][51]

The aforementioned single layer growth is also due to the low concentration of carbon in methane. Larger hydrocarbon gasses, such as ethane and propane, will lead to the growth of bilayer graphene.[52] In this light, it is obvious that at atmospheric-pressure CVD growth, multilayer graphene will also form on copper (similar to that grown on nickel films).[53] Growth of graphene has been demonstrated at temperatures compatible with conventional CMOS processing, using a nickel-based alloy with gold as catalysts.[54]

\section{GRAPHITE OXIDE REDUCTION}

Graphite oxide reduction was probably historically the first method of graphene synthesis. P. Boehm reported monolayer flakes of reduced graphene oxide already in 1962.[55] In this early work existence of monolayer reduced graphene oxide flakes was demonstrated. The contribution of Boehm was recently acknowledged by Nobel prize winner for graphene research, Andre Geim: (Many Pioneers in Graphene Discovery (http://www.aps.org/publications/apsnews/201001/letters.cf $\mathrm{m})$ ). Graphite oxide exfoliation can be achieved by

rapid heating and yields highly dispersed carbon powder with a few percent of graphene flakes. Reduction of graphite oxide monolayer films e.g. by hydrazine, annealing in argon/hydrogen was reported to yield graphene films. However, the quality of graphene produced by graphite oxide reduction is lower compared to e.g. 
scotch-tape graphene due to incomplete removal of various functional groups by existing reduction methods. Furhermore, the oxidation protocol introduces permanent defects due to over-oxidation. Recently, the oxidation protocol was essentially enhanced to yield graphene oxide with an almost intact carbon framework that allows the highly efficient removal of functional groups. The measured mobility of charge carriers exceeded 1000 $\mathrm{cm} 2 / \mathrm{Vs}$ for the best quality of flakes.[56] Some spectroscopic analysis of reduced graphene oxide can be found in the literature.[57] Applying a layer of graphite oxide film to a DVD disc and burning it in a DVD writer resulted in a thin graphene film with high electrical conductivity (1738 siemens per meter) and specific surface area (1520 square meters per gram), besides being highly resistant and malleable.[58][59] In February 2013 researchers from UCLA, led by professor Richard Kaner, announced a novel technique to produce graphene super capacitors based on the DVD burner approach.[60]

\section{GROWTH FROM METAL- CARBON MELTS}

The general idea in this process is to dissolve carbon atoms inside a transition metal melt at a certain temperature, and then allow the dissolved carbon to precipitate out at lower temperatures as single layer graphene (SLG).[61] The metal is first melted in contact with a carbon source. This source could be the graphite crucible inside which the melting process is carried out or it could be the graphite powder or chunk sources, which are simply placed in contact with the melt. Keeping the melt in contact with carbon source at a given temperature will give rise to dissolution and saturation of carbon atoms in the melt based on the binary phase diagram of metal-carbon. Upon lowering the temperature, solubility of the carbon in the molten metal will decrease and the excess amount of carbon will precipitate on top of the melt. The floating layer can be either skimmed or allowed to freeze for removal afterwards. Different morphology including thick graphite, few layer graphene (FLG) and SLG were observed on metal substrate. The Raman spectroscopy proved that SLG has been successfully grown on nickel substrate. The SLG Raman spectrum featured no $\mathrm{D}$ and $\mathrm{D}^{\prime}$ band, indicating the pristine and high-quality nature of SLG. Among transition metals, nickel provides a better substrate for growing SLG. Since nickel is not Raman active, the direct Raman spectroscopy of graphene layers on top of the nickel is achievable. The graphene-metal composite could be utilized in thermal interface materials for thermal management applications.[61] Pyrolysis of sodium ethoxide A 2008 publication described a process for producing gram-quantities of graphene, by the reduction of ethanol by sodium metal, followed by pyrolysis of the ethoxide product, and washing with water to remove sodium salts.[62]

\section{FROM NANOTUBES}

Experimental methods for the production of graphene ribbons are reported consisting of cutting open nanotubes.[63] In one such method multi-walled carbon nanotubes are cut open in solution by action of potassium permanganate and sulfuric acid.[64] In another method graphene nanoribbons are produced by plasma etching of nanotubes partly embedded in a polymer film.[65] From graphite by sonication It consists in dispersing graphite in a proper liquid medium that is then sonicated. Non exfoliated graphite is eventually separated from graphene by centrifugation. This method was first proposed by Hernandez et al.[66] who obtained graphene concentration up to $0.01 \mathrm{mg} / \mathrm{ml}$ in $\mathrm{N}$-methylpyrrolidone (NMP). The method was then largely improved by several groups, in particular, by the Italian group of Alberto Mariani. Mariani et al. reached the concentration of $2.1 \mathrm{mg} / \mathrm{ml}$ in NMP (the highest in this solvent).[67] The same group published the highest graphene concentrations reported so far in any liquid and obtained by any method. An example is the use of a suitable ionic liquid as the dispersing liquid medium for graphite exfoliation; [68] in this medium the very high concentration of $5.33 \mathrm{mg} / \mathrm{ml}$ was obtained. Carbon dioxide reduction method This synthesis process involves a highly exothermic reaction in which magnesium is combusted in an oxidation reduction reaction with carbon dioxide, producing a variety of carbon nanoparticles including graphene and fullerenes. The carbon dioxide reactant may be either solid (dry-ice) or gaseous. The products of this reaction are carbon and magnesium oxide. Issued patents are held on this process.[69][70]

\section{ATOMIC STRUCTURE}

The atomic structure of isolated, single-layer graphene was studied by transmission electron microscopy (TEM) on sheets of graphene suspended between bars of a metallic grid.[30] Electron diffraction patterns showed the expected honeycomb lattice of graphene. Suspended graphene also showed "rippling" of the flat sheet, with amplitude of about one nanometer. These ripples may be intrinsic to graphene as a result of the instability of two dimensional crystals,[2][71][72] or may be extrinsic, originating from the ubiquitous dirt seen in all TEM images of graphene. Atomic resolution real-space images of isolated, singlelayer graphene on $\mathrm{SiO} 2$ substrates were obtained[73][74] by scanning tunneling microscopy. Graphene processed using lithographic techniques is covered by photo resist residue, which must be cleaned to obtain atomic-resolution images.[73] Such residue may be the "adsorbates" observed in TEM images, and may explain the rippling of suspended graphene. Rippling of graphene on the $\mathrm{SiO} 2$ surface was determined to be caused by conformation of graphene to the underlying $\mathrm{SiO} 2$, and not an intrinsic effect.[73] Graphene sheets in solid form usually show evidence in diffraction for graphite's (002) layering. This is true even of some single-walled carbon nanostructures.[75] However, unlayered graphene with only (hk0) rings has been found in the core of presolar graphite onions.[76] TEM studies show faceting at defects in flat graphene sheets,[77] and suggest a possible role in this unlayered graphene for twodimensional crystallization from a melt. Graphene can selfrepair holes in its sheets, when exposed to molecules containing carbon, like hydrocarbons. When bombarded with pure carbon atoms, the holes in graphene sheets are completely filled, with carbon atom snapping to the gaps and perfectly aligning into hexagon shapes. [78][79]

\section{CONCLUSION}

In this study, we investigated the assembling, structure and mechanical properties of functionalized graphene materials. It is found that functional groups attached to a graphene surface can significantly affect its conformation by forming hydrogen bonds among these groups. With proper design of the amount and location of functional groups, folded structures with lower energies can be reached. Especially, a single hydrogen bond formed by two functional groups can 
International Research Journal of Engineering \& Applied Sciences, IRJEAS www.irjeas.org, ISSN(O): 2322-0821, Volume 9 Issue 3, Jul 2021-Sep 2021, Page 10-15

trigger an interesting self folding mechanism in which a graphene ribbon transforms into a double-helix form with a much larger persistence length (and changed mechanical properties). Folded graphene structures are stable and have the unfolding curves similar to what is known from biological protein molecules. We also studied the adhesion strength of the functionalized graphene interface and found that it is a monotonic function of the contacting length, which ensures its potential as a potent coating material.

It is noted that all the simulations in this study are based on the model of graphene functionalized by pure hydroxyl groups. This single functional group simplifies the modeling and design process because it can be both a donor and an acceptor in forming hydrogen bonds. There are other external factors, for example, the existence of the defects and dislocations within graphene or other functional groups like epoxy or carboxyl groups, which can affect the result. Further molecular dynamics simulations for much larger systems are needed to include those factors into the model, and to investigate the material morphology and mechanical properties.

Various protein materials have a universal backbone but have significant different mechanical properties [15]. For example, intermediate filament proteins are extensible and flexible at small strain, whereas spider silk proteins are less stretchable but feature a much higher strength. These mechanical characteristics are due to the fact that each material is encoded by chains of amino acids with different sequences made up from 20 different types of side chains that diversify interactions among amino acids, leading to various hierarchical conformations [30]. Graphene, as a low-dimensional material with a perfect lattice structure and extreme strength, is perfect to be used as the building block to form materials with great diversity, in analogy to protein materials.

The molecular structure of the graphene, including its geometry, chemical property and location of the functional groups, is important in affecting the morphology and material property of the structure, while the computational simulation show a significant advantage in facilitating this design work. By means of quantitative calculation and combining with experimental efforts, it may be possible to design and produce the next generation graphene-based materials. (Colour online) The size of the function graphene sheet is important in controlling the shearing and tearing strength of the material. (a): Simulation results of peak shearing forces and tearing forces before breaking apart of the graphene sheets, plotted as a function of the graphene length in contact. The shearing force is proportional to the contact area whereas the tearing force is proportional to the edge length as shown by different polynomial fitted curves. (b): Proposed set-up of a micro device coating method by using functionalized graphene sheets. $702 \mathrm{Z}$. Qin and M. Buehler

\section{REFERENCES}

[1] A.K. Geim and K.S. Novoselov, The rise of graphene, Nat. Mater. 6

(2007), pp. 183-191.
[2] J.C. Meyer, A.K. Geim, M.I. Katsnelson, K.S. Novoselov, T.J. Booth, and S. Roth, The structure of suspended graphene sheets, Nature 446 (2007), pp. 60-63.

[3] A.H. Castro Neto, F. Guinea, N.M.R. Peres, K.S. Novoselov, and A.K. Geim, The electronic properties of graphene, Rev. Mod. Phys. 81 (2009), pp. 109-162.

[4] A.A. Balandin, S. Ghosh, W.Z. Bao, I. Calizo, D. Teweldebrhan, F. Miao, and C.N. Lau, Superior thermal conductivity of single layer graphene, Nano Lett. 8 (2008), pp. 902-907.

[5] C. Lee, X.D. Wei, J.W. Kysar, and J. Hone, Measurement of the elastic properties and intrinsic strength of monolayer graphene, Science 321 (2008), pp. 385-388.

[6] A.K. Geim and P. Kim, Carbon wonderland, Sci. Am. 298 (2008), pp. 90-97.

[7] S. Bae, H. Kim, Y. Lee, X.F. Xu, J.S. Park, Y. Zheng, J. Balakrishnan, T. Lei, H.R. Kim, Y.I. Song, Y.J. Kim, K.S. Kim, B. Ozyilmaz, J.H. Ahn, B.H. Hong, and S. Iijima, Roll-to-roll production of 30-inch graphene films for transparent electrodes, Nat. Nanotech. 5 (2010), pp. 574-578.

[8] D. Sen, K.S. Novoselov, P.M. Reis, and M.J. Buehler, Tearing graphene sheets from adhesive substrates produces tapered nanoribbons, Small 6 (2010), pp. 1108-1116.

[9] N.V. Medhekar, A. Ramasubramaniam, R.S. Ruoff, and V.B. Shenoy, Hydrogen bond networks in graphene oxide composite paper: Structure and mechanical properties, ACS Nano 4 (2010), pp. 2300-2306.

[10] D.R. Paul, Creating new types of carbon-based membranes, Science 335 (2012), pp. 413-414. Figure 6.

[11] R.R. Nair, H.A. Wu, P.N. Jayaram, I.V. Grigorieva, and A.K. Geim, Unimpeded permeation of water through helium-leak-tight graphene- based membranes, Science 335 (2012), pp. 442-444.

[12] A.V. Finkelstein, O.V. Galzitskaya, and A.Y. Badretdinov, A folding pathway solving Levinthal's paradox, Prog. Biophys. Mol. Biol. 65 (1996), pp. 53-53.

[13] A. Sali, E. Shakhnovich, and M. Karplus, How does a protein fold, Nature 369 (1994), pp. 248-251.

[14] M.J. Buehler, and S. Keten, Strength and resilience: A comparative study on mechanical signatures of alpha-helix, betasheet and tropocollagen domains, Nano Res. 1 (2008), pp. 63-71.

[15] M.J. Buehler, Turning weakness to strength, Nano Today 5 (2010), pp. 379-383.

[16] S.J. Stuart, A.B. Tutein, and J.A. Harrison, A reactive potential for hydrocarbons with intermolecular interactions, J. Chem. Phys. 112 (2000), pp. 6472-6486.

[17] D.W. Brenner, O.A. Shenderova, J.A. Harrison, S.J. Stuart, B. $\mathrm{Ni}$, and S.B. Sinnott, A second-generation reactive empirical bond order (REBO) potential energy expression for hydrocarbons, J. Phys.-Condens. Matter. 14 (2002), pp. 783-802.

[18] A.D. MacKerell, D. Bashford, M. Bellott, R.L. Dunbrack, J.D. Evanseck, M.J. Field, S. Fischer, J. Gao, H. Guo, S. Ha, D. JosephMcCarthy, L. Kuchnir, K. Kuczera, F.T.K. Lau, C. Mattos, S. Michnick, T. Ngo, D.T. Nguyen, B. Prodhom, W.E. Reiher, B. Roux, M. Schlenkrich, J.C. Smith, R. Stote, J. Straub, M. Watanabe, J. Wiorkiewicz-Kuczera, D. Yin, and M. Karplus, Allatom empirical potential for molecular modeling and dynamics studies of proteins, J. Phys. Chem. B 102 (1998), pp. 3586-3616. 
International Research Journal of Engineering \& Applied Sciences, IRJEAS

www.irjeas.org, ISSN(O): 2322-0821, Volume 9 Issue 3, Jul 2021-Sep 2021, Page 10-15

[19] Q.B. Zheng, Y. Geng, S.J. Wang, Z.G. Li, and J.K. Kim, Effects of functional groups on the mechanical and wrinkling properties of graphene sheets, Carbon 48 (2010), pp. 4315-4322.

[20] A.F. Fonseca, G. Lee, T.L. Borders, H.J. Zhang, T.W. Kemper, T.R. Shan, S.B. Sinnott, and K. Cho, Reparameterization of the REBO-CHO potential for graphene oxide molecular dynamics simulations, Phys. Rev. B 84 (2011), 075460.

[21] S. Keten, Z.P. Xu, B. Ihle, and M.J. Buehler, Nano confinement controls stiffness, strength and mechanical toughness of beta-sheet crystals in silk, Nat. Mater. 9 (2010), pp. 359-367.

[22] Z. Qin and M.J. Buehler, Molecular dynamics simulation of the alpha-helix to beta-sheet transition in coiled protein filaments: Evidence for a critical filament length scale, Phys. Rev. Lett. 104 (2010), 198304.

[23] Z. Qin, L. Kreplak, and M.J. Buehler, Hierarchical structure controls nanomechanical properties of vimentin intermediate filaments, PLoS ONE 4 (2009), e7294.

[24] Z.P. Xu, and M.J. Buehler, Geometry controls conformation of graphene sheets: Membranes, ribbons, and scrolls, ACS Nano 4 (2010), pp. 3869-3876.

[25] F. Grater, P. Heider, R. Zangi, and B.J. Berne, Dissecting entropic coiling and poor solvent effects in protein collapse, J. Am. Chem. Soc. 130 (2008), pp. 11578-11579.

[26] J.F. Marko, and E.D. Siggia, Stretching DNA, Macromolecules 28 (1995), pp. 8759-8770.

[27] S. Park, and R.S. Ruoff, Chemical methods for the production of graphenes, Nat. Nanotech. 4 (2009), pp. 217-224.

[28] S. Keten, and M.J. Buehler, Geometric confinement governs the rupture strength of H-bond assemblies at a critical length scale, Nano Lett. 8 (2008), pp. 743-748.

[29] Z. Qin, and M.J. Buehler, Cooperative deformation of hydrogen bonds in beta-strands and beta-sheet nanocrystals, Phys. Rev. E 82 (2010), 061906.

[30] M.J. Buehler, Strength in numbers, Nat. Nanotech. 5 (2010),pp. 172-174. 